\title{
ITERATIVE SOLUTION OF A WIENER-HOPF PROBLEM IN SEVERAL VARIABLES ${ }^{1}$
}

\section{STAVROS N. BUSENBERG}

Abstract. An extension of the classical problem of Wiener and Hopf to functions of several complex variables is considered. A sufficient condition for the unique solvability of the problem is obtained. Finally, a method for an iterative construction of the solution is given.

1. Introduction. A recent analysis of a diffraction problem has led Kraut and Lehman [1] to extend a classical problem of Wiener and Hopf [2], [3] to functions of several complex variables. To pose the problem let $R^{n}$ be the real $n$-space and $C^{n}$ the space of $n$ complex variables $z=\left(z_{1}, \cdots, z_{n}\right), z_{j}=x_{j}+i y_{j}$. Let $S_{0} \subset R^{n}$ be the set $\left\{y=\left(y_{1}, \cdots, y_{n}\right): \gamma_{j}<y_{j}<\delta_{j}\right\}$, and by the tube $T_{0}$ with the basis $S_{0}$ denote the set $\left\{z=x+i y \in C^{n}: y \in S_{0}\right\}$. To each of the $2^{n}$ possible choices of $y_{j}>\gamma_{j}$ or $y_{j}<\delta_{j}$ attach basis sets $S_{p}$ and corresponding tubes $T_{p}$, $p=1, \cdots, 2^{n}$, in some order, such that $S_{1}=\left\{y: \gamma_{j}<y_{j}, j=1, \cdots, n\right\}$. By $A\left(T_{p}\right)$ denote the class of functions $f: T_{p} \rightarrow C$ which are analytic on $T_{p}$, and by $A_{0}\left(T_{p}\right)$ denote all $f \in A\left(T_{p}\right), p \neq 0$, obeying $f(z) \rightarrow 0$ as any $\left|y_{j}\right| \rightarrow \infty$ in $S_{p}$. Finally, by $H\left(T_{p}\right)$ denote the class of all $f \in A\left(T_{p}\right)$ such that

$$
\|f\|_{p}=\sup _{y \in S_{p}}\left\{\int \cdots \int|f(z)|_{-\infty}^{+\infty} d x_{1} \cdots d x_{n}\right\}^{1 / 2}<\infty .
$$

The extended Weiner-Hopf problem (henceforth abbreviated to the EWH problem) can be stated as follows: Given a function $k \in A\left(T_{0}\right)$ and bounded on $T_{0}$ and given $g_{1} \in H\left(T_{1}\right)$, find $2^{n}$ functions $f_{p} \in H\left(T_{p}\right)$, $p=1, \cdots, 2^{n}$, such that

$$
k(z) f_{1}(z)=g_{1}(z)+\sum_{p=2}^{2^{n}} f_{p}(z), \quad \forall z \in T_{0} .
$$

2. The main results. This section is devoted to the proof of Theorems 1 and 2 below.

Presented to the Society, January 22, 1970 under the title $A$ Wiener-Hopf problem in several variables; received by the editors January $12,1970$.

AMS 1969 subject classifications. Primary 3215, 4240; Secondary 4515, 7845.

Key words and phrases. Multidimensional Wiener-Hopf problem, multiple Fourier transforms, tubular domains, electromagnetic diffraction, contraction mapping.

1 This research was supported in part by National Science Foundation grant GP-12407.

Copyright (1) 1971. American Mathematical Society 
THEOREM 1. If the range of $k$ for $z \in T_{0}$ lies in a closed, bounded, convex set which does not contain the origin, then the EWH problem has a unique solution.

It is noted that under the conditions of Theorem 1, $\exists$ a closed disk in $C$ which contains the range of $k$ and does not contain the origin. Let $w \neq 0$ be the center of this disk, and note that its radius is less than $|w|$. Thus, if

$$
h(z)=k(z)-w, \quad z \in T_{0},
$$

it follows that $h(z) \in A\left(T_{0}\right)$, is bounded on $T_{0}$, and

$$
\sup _{z \in T_{0}}(|h(z)|)<|w| \text {. }
$$

Next, introduce the notation $s\left(T_{p}\right)$ to denote the spine of $T_{p}, p \neq 0$. That is, if $S_{p}$ is given by inequalities of the forms $\gamma_{j}<y_{j}$ or $y_{j}<\delta_{j}$, denoting by $\partial S_{p}^{j}$ the lines $\left(-\infty+i \gamma_{j}, \infty+i \gamma_{j}\right)$ or $\left(\infty+i \delta_{j},-\infty+i \delta_{j}\right)$, respectively, then $s\left(T_{p}\right)=\prod_{j=1}^{n} \partial S_{p}^{j}$.

Finally, if $f \in H\left(T_{p}\right), p \neq 0$, note that (Bochner [4]) the Cauchy integral formula

$$
f(z)=(2 \pi i)^{-n} \int_{s\left(T_{p}\right)} \ldots \int f(\zeta) \prod_{j=1}^{n}\left(\zeta_{j}-z_{j}\right)^{-1} d \zeta_{j}, \quad z \in T_{p},
$$

is valid, where the boundary value $f(\zeta)$ of $f(z)$ is square integrable over $R^{n}$ as a function of $x$, and is attained a.e. as $z \rightarrow \zeta \in s\left(T_{p}\right), z \in T_{p}$.

THEOREM 2. Let $k$ satisfy the conditions of Theorem 1 , and let $w \in C$ and $h(z)$ be given as in (3). Define the operator $L: H\left(T_{0}\right) \rightarrow H\left(T_{1}\right)$ by

$$
L(f)(z)=w^{-1}\left[g_{1}(z)-(2 \pi i)^{-n} \int_{z\left(T_{1}\right)} \ldots \int h(\zeta) f(\zeta) \prod_{j=1}^{n}\left(\zeta_{j}-z_{j}\right)^{-1} d \zeta_{j}\right],
$$

$$
f \in H\left(T_{0}\right) \text {. }
$$

Then the sequence $f_{1}^{j}$ given by

$$
f_{1}^{0}=g_{1}, \quad f_{1}^{j}=L\left[f_{1}^{j-1}\right],
$$

converges uniformly on compact subsets of $T_{1}$ to the $f_{1}$ of the solution $\left\{f_{p}\right\}_{p=1}^{2^{n}}$ of the EWH problem. The remaining $f_{p}, p \neq 1$, are given by

$$
f_{p}(z)=(2 \pi i)^{-n} \int_{z\left(T_{p}\right)} \ldots \int\left[k(\zeta) f_{1}(\zeta)-g_{1}(\zeta)\right] \prod_{j=1}^{n}\left(\zeta_{j}-z_{j}\right)^{-1} d \zeta_{j}
$$


Before proceeding with the proofs of these theorems, we present five lemmas, some of which are in the literature, but are listed here for ease of future reference. The first is a special case of a theorem of Bochner [5].

Lемма 1. $F \in H\left(T_{p}\right)$ for some $p$, iff $\exists a$ measurable function $\phi(t)=\phi\left(t_{1}, \cdots, t_{n}\right)$ on $R^{n}$ such that

$$
\phi_{y}(t)=\phi(t) \exp \left(-\sum_{j=1}^{n} y_{j} t_{j}\right) \in L^{2}\left(R^{n}\right), \quad \forall y \in S_{p},
$$

(i.e., $\phi_{y}(t)$ is square integrable) and

$$
F(z)=\int_{-\infty}^{\infty} \cdots \int \exp \left(\sum_{j=1}^{n} i z_{j} t_{j}\right) \phi(t) d t_{1} \cdots d t_{n}, \quad z \in T_{p} .
$$

Moreover,

$$
\|F\|_{p}=\sup _{\nu \in S_{p}}\left((2 \pi)^{n / 2} \int \cdots \int\left|\phi_{-\infty}^{\infty}(t)\right|^{2} d t_{1} \cdots d t_{n}\right)^{1 / 2} .
$$

For the proof see [5] or else Bochner and Martin [6]. The next result is essentially also due to Bochner [5].

Lemma 2. If $F \in H\left(T_{0}\right), \exists 1$ set $\left\{F_{p}\right\}_{p=1}^{2^{n}}$ of functions $F_{p} \in A_{0}\left(T_{p}\right)_{3}$

$$
F(z)=\sum_{p=1}^{2^{n}} F_{p}(z), \quad z \in T_{0},
$$

and

$$
F_{p}(z)=L_{p}[F](z)=(2 \pi i)^{-n} \int_{s\left(T_{p}\right)} \ldots \int F(\zeta) \prod_{j=1}^{n}\left(\zeta_{j}-z_{j}\right)^{-1} d \zeta_{j}
$$

$$
z \in T_{p}
$$

For the proof see Bochner [5], and for the uniqueness part of the result see Kraut et al. [7].

Lemma 3. If $f_{p} \in H\left(T_{p}\right)$ for some $p=1, \cdots, 2^{n}$, then $f_{p} \in A_{0}\left(T_{p}\right)$. Moreover, if $f_{p}^{j} \in H\left(T_{p}\right), j=1,2, \cdots$, and if $\left\|f_{p}^{j}-f_{p}\right\|_{p} \rightarrow 0$, as $j \rightarrow \infty$, then $f_{p}^{j} \rightarrow f_{p}(z)$ uniformly on compact subsets of $T_{p}$.

Proof. If $z^{0} \in T_{p}$ and if $l_{p} \in H\left(T_{p}\right)$, then $l_{p}$ can be expressed as a convergent Taylor series in a polydisk $K$ with center $z^{0}$ and nonzero radius $r=\left(r_{1}, \cdots, r_{n}\right)$. Moreover, $r_{j}=y_{j}^{0}-\gamma_{j}$ or $r_{j}=\delta_{j}-y_{j}^{0}$, are the greatest possible radii of $K$. Thus 


$$
\begin{aligned}
& \int \cdots \int_{K}\left|l_{p}(z)\right|^{2} \prod_{j=1}^{n} d x_{j} d y_{j} \\
& =\int \cdots \cdot \underset{0}{2 \pi} \iint_{0}^{r_{1}} \int_{0}^{r_{n}}\left|\sum_{|\alpha|=0}^{\infty} a_{\alpha} \exp \left(i \sum_{j=1}^{n} \alpha_{j} \theta_{j}\right) \prod_{j=1}^{n} r_{j}^{\alpha_{j}}\right|^{2} \prod_{j=1}^{n} r_{j} d r_{j} d \theta_{j}
\end{aligned}
$$

$$
\begin{aligned}
& =(2 \pi)^{n} \sum_{|\alpha|}\left|a_{\alpha}\right|^{2} \prod_{j=1}^{n} r_{j}^{2 \alpha_{j}+2}\left(2 \alpha_{j}+2\right)^{-1} \\
& \geqq \prod_{j=1}^{n} \pi r_{j}^{2}\left|l_{p}\left(z^{0}\right)\right|^{2}
\end{aligned}
$$

where $\alpha=\left(\alpha_{1}, \cdots, \alpha_{n}\right),|\alpha|=\sum_{j=1}^{n} \alpha_{j}$; and $a_{0}=l\left(z^{0}\right)$ was used in the last step. Now

$$
\int \cdots \int_{K}\left|l_{p}(z)\right|^{2} d x_{1} d y_{1} \cdots d x_{n} d y_{n} \leqq 2^{n}\left\|l_{p}\right\|_{p}^{2} \prod_{j=1}^{n} r_{j} .
$$

Combining (13) and (14) it is seen that

$$
\left|l_{p}\left(z^{0}\right)\right| \leqq\left(2 \pi^{-1}\right)^{n / 2} \prod_{j=1}^{n} r_{j}^{-1 / 2}\left\|l_{p}\right\|_{p}
$$

Hence, as any $\left|y_{j}\right| \rightarrow \infty$ in $T_{p}$ it is seen that $r_{j} \rightarrow \infty$ and $l_{p}\left(z^{0}\right) \rightarrow 0 \Rightarrow l_{p}$ $\in A_{0}\left(T_{p}\right)$, and the first part of the lemma is established.

Next, if $U$ is a compact subset of $T_{p}$, then the distance from $U$ to $\partial T_{p}$ is positive; hence, $\exists \beta_{j} \ni 0<\beta_{j}<r_{j}, j=1, \cdots, n$, holds $\forall z^{0} \in U$. Thus

$$
\left|l_{p}\left(z^{0}\right)\right| \leqq\left(2 \pi^{-1}\right)^{n / 2} \prod_{j=1}^{n} \beta_{j}^{-1 / 2}\left\|l_{p}\right\|_{p}, \quad \forall z^{0} \in U
$$

and convergence in the $\|\cdot\|_{p}$ norm implies uniform convergence in $U$. This completes the proof of the lemma.

Lemma 4. If $F \in H\left(T_{0}\right), \exists 1$ set $\left\{F_{p}\right\}=\left\{L_{p}[F]\right\}, p=1, \cdots, 2^{n}$, of functions $F_{p} \in H\left(T_{p}\right)_{3}$

$$
F(z)=\sum_{p=1}^{2^{n}} F_{p}(z)=\sum_{p=1}^{2^{n}} L_{p}[F](z), \quad z \in T_{0},
$$

where the $L_{p}$ are defined by (12), and

$$
\left\|L_{p}[F]\right\|_{0} \leqq\|F\|_{0}, \quad p=1, \cdots, 2^{n} .
$$


Proof. Only the existence of the $F_{p} \in H\left(T_{p}\right)$, and hence $F_{p} \in A_{0}\left(T_{p}\right)$ by Lemma 3, satisfying the first part of (17) and (18) need be shown, since uniqueness, and thence, the remainder of (17) will follow from Lemma 2.

From Lemma $1 \exists \phi(t)$ measurable in $R^{n}{ }$ (8) and (9) hold with $p=0$. The integral in (9) may be written as the sum of $2^{n}$ integrals obtained by writing each of the single integrals as the sum of an integral on $(-\infty, 0)$ and one on $(0, \infty)$. With each $S_{p}$ associate one of these $2^{n}$ integrals, denoted by $\left(\int \cdots \int\right)_{p}$, as follows: whenever $y_{j}>\gamma_{j}\left(y_{j}<\delta_{j}\right)$ enters in the definition of $S_{p}$, the $j$ th variable is integrated on $(0, \infty)((-\infty, 0))$. Then

$$
F(z)=\sum_{p=1}^{2^{n}}\left(\int \cdots \int\right)_{p} \exp \left(\sum_{j=1}^{n} i z_{j} t_{j}\right) \phi(t) d t_{1} \cdots d t_{n},
$$

$$
z \in T_{0}
$$

It will now be shown that the first integral $(p=1)$ yields the $F_{1}$ in (17).

From (8) and Fubini's theorem $\int_{-\infty}^{\infty} \exp \left(-2 y_{1} t_{1}\right)|\phi(t)|^{2} d t_{1}$ exists for $\gamma_{1}<y_{1}<\delta_{1}$ for almost all $t^{\prime}=\left(t_{2}, \cdots, t_{n}\right) \in R^{n-1}$. Hence, $\int_{0}^{\infty} \exp \left(-2 y_{1} t_{1}\right)|\phi(t)|^{2} d t_{1}$ converges for $\gamma_{1}<y_{1}$, and

$$
\exp \left(-\sum_{j=2}^{n} 2 y_{j} t_{j}\right) \int_{0}^{\infty} \exp \left(-2 y_{1} t_{1}\right)|\phi(t)|^{2} d t_{1}
$$

is integrable for $t^{\prime} \in R^{n-1}$ and for $\gamma_{j}<y_{j}<\delta_{j}, j=2, \cdots, n$. Repeating the above argument for the remaining $(n-1)$ variables, it follows that $\left(\int \cdots \int\right)_{1}\left(-\sum_{j=1}^{n} 2 y_{j} t_{j}\right)|\phi(t)|^{2} \prod_{m=1}^{n} d t_{m}$ converges for $y \in S_{1}$. Defining $\phi(t)$ to be zero when $y_{j}<0$ and using Lemma 1 , it is seen that the first term in (19) belongs to $H\left(T_{1}\right)$, and may be set equal to $F_{1}$. Applying the same argument to the remaining integrals for $p$ $=2, \cdots, 2^{n}$, in (19), the first part of (17) is established.

From (10) of Lemma 1 it is seen that

$$
\begin{aligned}
\left\|F_{p}\right\|_{0}^{2} & =\sup _{\nu \in S_{0}}(2 \pi)^{n / 2}\left(\int \cdots \int\right)_{p}\left|\phi_{y}(t)\right|^{2} d t_{1} \cdots d t_{n} \\
& \leqq \sup _{\nu \in S_{0}}(2 \pi)^{n / 2} \int \cdots{ }_{-\infty}^{\infty} \int\left|\phi_{\nu}(t)\right|^{2} d t_{1} \cdots d t_{n}=\|F\|_{0 .}^{2}
\end{aligned}
$$

This completes the proof of Lemma 4. 
Lemma 5. If $f_{1} \in H\left(T_{1}\right)$ then

$$
\left\|f_{1}\right\|_{1}=\left\|f_{1}\right\|_{0}=\lim _{y \rightarrow \gamma}\left\{\int \ldots \int|f(z)|^{2} d x_{1} \cdots d x_{n}\right\}^{1 / 2} .
$$

Proof. Let

$$
\begin{aligned}
& M_{1}(y ; f)=\left\{\int_{-\infty}^{\infty}|f(z)|^{2} d x_{1}\right\}^{1 / 2}, \\
& M(y ; f)=\left\{\int_{-\infty}^{\infty} \ldots \int|f(z)|^{2} d x_{1} \cdots d x_{n}\right\}^{1 / 2} .
\end{aligned}
$$

It is known (see Hille [8]) that $M_{1}(y ; f)$ is a nonincreasing function of $y_{1}$. Now, if $b_{1}<y_{1}$,

$$
\begin{aligned}
\int \cdots \int\left[M_{1}(y ; f)\right]^{2} d x_{2} \cdots d x_{n} \\
\\
\qquad \int_{-\infty}^{\infty} \cdots \int\left[M_{1}\left(b_{1}, y_{2}, \cdots, y_{n} ; f\right)\right]^{2} d x_{2} \cdots d x_{n} .
\end{aligned}
$$

Thus, $M(y ; f) \leqq M\left(b_{1}, y_{2}, \cdots, y_{n}, f\right)$.

In a similar way, it can be shown that $M(y ; f)$ is a nonincreasing function of $y_{j}, j=2, \cdots, n$. This fact and the definition (1) of $\|\cdot\|_{0}$ and $\|\cdot\|_{1}$ establish the lemma.

Proof of TheOREM 1. It is first noted that $H\left(T_{0}\right)$ is a linear space with scalars in $C$ and that $\|\cdot\|_{0}$ is a norm over this space. It follows readily, using Lemma 3 (see [6, Chapter VI] for example) that $H\left(T_{0}\right)$ is a Banach space.

Consider the operator defined by (5) and use the notation in (12) to obtain

$$
L[f]=w^{-1}\left(g_{1}-L_{1}[h f]\right), \quad f \in H\left(T_{0}\right) .
$$

Since $h$ is bounded and $h \in A\left(T_{0}\right), h f \in H\left(T_{0}\right)$ and, by Lemma 4, $L_{1}[h f] \in H\left(T_{1}\right)$. Thus $L$ is a linear operator.

$$
L: H\left(T_{0}\right) \rightarrow H\left(T_{1}\right) \subset H\left(T_{0}\right) .
$$

From (22), the linearity of $L_{1}$ and Lemma 4, it is seen that if $F$ and $G \in H\left(T_{0}\right)$,

$$
\|L[F]-L[G]\|_{0}=|w|^{-1}\left\|L_{1}[h(F-G)]\right\|_{0} \leqq|w|^{-1}\|h(F-G)\|_{0} .
$$

Now, from (1), (4) and (24),

$$
\|L[F]-L[G]\|_{0}<|w|^{-1} \sup _{z \in T_{0}}|h(z)|\|F-G\|_{0}<\alpha\|F-G\|_{0}
$$


where $0<\alpha<1$. Thus $L$ is a contraction mapping and $\exists 1 f \in H\left(T_{0}\right)$ $\rightarrow L[f]=f$. But, from (23), it follows that $f=f_{1} \in H\left(T_{1}\right)$, and hence $\exists 1 f_{1} \in H\left(T_{1}\right)$ э

$$
L\left[f_{1}\right]=f_{1} .
$$

From (3), (5) and (26), it is seen that $w f_{1}=g_{1}-L_{1}\left[(k-w) f_{1}\right]$, whence, using (26) again, it follows that

$$
L_{1}\left[k f_{1}\right]=g_{1} \text {. }
$$

However, $k f_{1} \in H\left(T_{0}\right)$ and by Lemma 4 and (27), $\exists 1$ set $\left\{L_{p}\left[k f_{1}\right]\right\}_{p=1}^{2^{n}}, L_{p}\left[k f_{1}\right] \in H\left(T_{p}\right)$ ?

$$
k f_{1}(z)=\sum_{p=1}^{2^{n}} L_{p}\left[k f_{1}\right](z)=g_{1}(z)+\sum_{p=2}^{2^{n}} L_{p}\left[k f_{1}\right](z), \quad z \in T_{0} .
$$

This completes the proof of Theorem 1.

Proof of Theorem 2. From the proof of Theorem 1, it follows that the sequence $f_{1}^{j}, j=0,1, \cdots$, defined in (6) converges in the $\|\cdot\|_{0}$ norm to the $f_{1}$ of the solution $\left\{f_{p}\right\}_{p=1}^{2^{n}}$ of the EWH problem. Moreover, $f_{1}^{j} \in H_{1}\left(T_{1}\right) \forall j$, and by Lemma $5, f_{1}^{j} \rightarrow f_{1}$ in the $\|\cdot\|_{1}$ norm and hence uniformly in compact subsets of $T_{1}$. This establishes the first part of Theorem 2. To complete the proof, note that equation (7) is a consequence of equation (28) and Lemma 4.

3. Remarks on previous results. As a special case of Theorem 1 it is noted that if $k(z)$ obeys

$$
|1-k(z)|<1, \quad \forall z \in T_{0},
$$

then the EWH problem is uniquely solvable. Kraut [9] and Kraut and Lehman [1] considered the case $\gamma_{j}<0<\delta_{j}, j=1, \cdots, n$, and the class of kernels $k(z)=1-\lambda h(z)$, where $\lambda$ is a real parameter which can be varied. They replace the condition (29) by the weaker condition $|1-k(z)|<1, z_{j}=x_{j}, j=1, \cdots, n$. This latter condition is not sufficient for the unique solvability of the EWH problem, as is seen by taking (for $n=2), \quad k(z)=1+\left[2\left(z_{1}+i\right)\left(z_{2}+i\right)\right]^{-1}, \quad g(z)=\left(z_{1}+i\right)^{-1}$ $\cdot\left(z_{2}+i\right)^{-1}$, and $T_{0}=\left\{z \in C^{2}:-3 / 4<y_{j}<3 / 4\right\}$. The resulting EWH problem satisfies this latter condition but has no solution. However, since the $h(z)$ in [1] and [9] is uniformly bounded in $T_{0}, \lambda$ can be chosen small enough for (29) to hold in $T_{0}$, and from Theorem 1 the resulting EWH problem has a unique solution.

The author was introduced to this problem by Dr. Edgar Kraut. $\mathrm{He}$ is indebted to him as well as to Professor Robert Borrelli and Dr. William Hall for a number of discussions and suggestions. 


\section{REFERENCES}

1. E. A. Kraut and G. W. Lehman, Diffraction of electromagnetic waves by a rightangle dielectric wedge, J. Mathematical Phys. 10 (1969), 1340-1348. MR 40 \#2285.

2. N. Wiener and E. Hopf, Über eine Klasse singularer Integralgleichungen, S.-B. Preuss. Akad. Wiss. 1931, 696-706.

3. M. G. KreIn, Integral equations on a half-line with kernel depending upon the difference of the arguments, Uspehi Mat. Nauk 13 (1958), no. 5 (83), 3-120; English transl., Amer. Math. Soc. Transl. (2) 22 (1962), 163-288. MR 21 \#1507.

4. S. Bochner, Group invariance of Cauchy's formula in several variables, Ann. of Math. (2) 45 (1944), 686-707. MR 6, 123.

5. - Bounded analytic functions in several variables and multiple Laplace integrals, Amer. J. Math. 59 (1937), 732-738.

6. S. Bochner and W. T. Martin, Several complex variables, Princeton Univ. Press, Princeton, N. J., 1948. MR 10, 366.

7. E. Kraut, S. Busenberg and W. Hall, On an additive decomposition of functions of several complex variables, Bull. Amer. Math. Soc. 74 (1968), 372-374. MR 36 \#2826.

8. E. Hille, Analytic function theory. Vol. II, Ginn, Boston, Mass., 1962. MR 34 \#1490.

9. E. Kraut, On equations of the Wiener-Hopf type in several complex variables, Proc. Amer. Math. Soc. 23 (1969), 24-26.

Harvey Mudd College, Claremont, California 91711 